ESAIM: M2AN

Vol. 41, No 5, 2007, pp. 855-874

DOI: $10.1051 / \mathrm{m} 2 \mathrm{an}: 2007034$
ESAIM: Mathematical Modelling and Numerical Analysis

www.esaim-m2an.org

\title{
INF-SUP STABLE NONCONFORMING FINITE ELEMENTS OF HIGHER ORDER ON QUADRILATERALS AND HEXAHEDRA
}

\author{
Gunar Matthies ${ }^{1}$
}

\begin{abstract}
We present families of scalar nonconforming finite elements of arbitrary order $r \geq 1$ with optimal approximation properties on quadrilaterals and hexahedra. Their vector-valued versions together with a discontinuous pressure approximation of order $r-1$ form inf-sup stable finite element pairs of order $r$ for the Stokes problem. The well-known elements by Rannacher and Turek are recovered in the case $r=1$. A numerical comparison between conforming and nonconforming discretisations will be given. Since higher order nonconforming discretisation on quadrilaterals and hexahedra have less unknowns and much less non-zero matrix entries compared to corresponding conforming methods, these methods are attractive for numerical simulations.
\end{abstract}

Mathematics Subject Classification. 65N12, 65N30.

Received October 25, 2005.

\section{Introduction}

The spaces $Q_{r}, r \geq 1$, of continuous, piecewise polynomials of degree $r$ in each variable on quadrilaterals and hexahedra form families of finite elements of optimal order $r$ with respect to the $H^{1}$-norm if scalar elliptic problems are considered. The situation is much less clear if we look for families of nonconforming pairs of order $r$. The use of nonconforming approximations is attractive since their degrees of freedom are edge-oriented on quadrilaterals and face-oriented on hexahedra, respectively. This results in cheap communication when these methods are implemented on a parallel computer.

Nonconforming discretisations of higher order on quadrilaterals and hexahedra have less degrees of freedom and much less non-zero matrix entries than the corresponding conforming discretisations. Indeed, we have for the conforming $Q_{r}$-element on regular $N \times N$ decompositions of the unit square approximately $N^{2} r^{2}$ degrees of freedom and $N^{2} r^{4}$ non-zero matrix entries. Our nonconforming elements of order $r$ will result in approximately $N^{2} r^{2} / 2$ degrees of freedom and $N^{2} r^{4} / 4$ non-zero matrix entries. The behaviour will be more dramatic in three space dimensions. We have for the conforming $Q_{r}$-element on regular $N \times N \times N$ decompositions of the unit cube approximately $N^{3} r^{3}$ degrees of freedom and $N^{3} r^{6}$ non-zero matrix entries. Our nonconforming element of order $r$ will result in approximately $N^{3} r^{6} / 6$ degrees of freedom and $N^{3} r^{3} / 36$ non-zero matrix entries. This makes nonconforming discretisations attractive form the practical point of view.

Nonconforming finite element methods can be regarded as mortar finite element methods on element level where the additional Lagrangian multipliers have been eliminated. Moreover, nonconforming finite element

Keywords and phrases. Nonconforming finite elements, inf-sup stability, quadrilaterals, hexahedra.

1 Fakultät für Mathematik, Ruhr-Universität Bochum, Universitätsstraße 150, 44780 Bochum, Germany.

Gunar.Matthies@ruhr-uni-bochum.de

(c) EDP Sciences, SMAI 2007 Article published by EDP Sciences and available at http://www.esaim-m2an.org or http://dx.doi.org/10.1051/m2an:2007034 
methods can be seen somehow between conforming finite element methods and discontinuous Galerkin methods. The continuity requirement of conforming finite element methods is weakened in nonconforming finite element methods but not removed completely from the approximation spaces as it is done in discontinuous Galerkin methods. Furthermore, the use of nonconforming finite element methods avoids the implementation of jumps terms which are essential for discontinuous Galerkin methods.

We consider the stationary Stokes problem in the following form

$$
\begin{aligned}
-\triangle \boldsymbol{u}+\nabla p=\boldsymbol{f} & \text { in } \Omega, \\
\operatorname{div} \boldsymbol{u}=0 & \text { in } \Omega, \\
\boldsymbol{u}=\boldsymbol{g} & \text { on } \partial \Omega .
\end{aligned}
$$

Here, $\Omega \subset \mathbb{R}^{d}, d=2,3$, is a domain, $\boldsymbol{f}$ and $\boldsymbol{g}$ are given sufficiently smooth functions. Furthermore, we assume that the compatibility condition

$$
\int_{\partial \Omega} \boldsymbol{g} \cdot \boldsymbol{n} \mathrm{d} \gamma=0
$$

is fulfilled where $\boldsymbol{n}$ denotes the outer unit normal vector on $\partial \Omega$.

On quadrilaterals and hexahedra, there are two widely used families of conforming finite element pairs of arbitrary order for approximating velocity and pressure in the Stokes problem. First, we discuss the Taylor Hood family where the pairs $Q_{r} / Q_{r-1}, r \geq 2$, of continuous velocity and continuous pressure approximations are used. For the proof of the inf-sup condition for these pairs, we refer to [1,9,22]. For getting a mass conservation on element level, discontinuous pressure approximations are advantageous. On both quadrilaterals and hexahedra, the family $Q_{r} / P_{r-1}^{\text {disc }}, r \geq 2$, satisfies the inf-sup condition, see $[9,16]$.

Due to their advantages in parallelisation and their reduced number of unknowns and non-zero matrix entries, we consider nonconforming velocity approximations. A general theory for analysing nonconforming methods applied to saddle point problems can be found in [6].

To the author's knowledge, only lowest order nonconforming methods on quadrilaterals and hexahedra have been considered so far, see $[4,5,7,10,19]$. One reason could be that there seems to be no generic construction for a family of nonconforming discretisations which is suited for the Stokes problem. Nonconforming finite elements of higher order on triangles were considered in $[17,18]$ while only [17] focuses on their application to the Stokes problem.

We will consider in this paper four families of scalar nonconforming finite elements, three on quadrilaterals and one on hexahedra. The case $r=1$ recovers for all four families the well-known rotated multi-linear elements by Rannacher and Turek [19]. It will be shown that the vector-valued version of the element of order $r$ for the velocity approximation will be inf-sup stable together with a discontinuous piecewise $P_{r-1}$ approximation of the pressure. One of the scalar families on quadrilaterals and the scalar family on hexahedra can be already found in [11] but their consideration for approximating the Stokes problem (1) is new. We show for the two novel family on quadrilaterals their general construction principles and prove their unisolvence with respect to the nodal functionals. Furthermore, a comparison between the nonconforming families and the well-known conforming families $Q_{r} / Q_{r-1}$ and $Q_{r} / P_{r-1}^{\text {disc }}$ with respect to the number of degrees of freedom, the number of non-zero matrix entries, the accuracy, and the orders of convergence will be given.

Since we are mainly interested in deriving novel finite elements, we consider in this paper for simplicity only the case where all cells are affine equivalent to the unit square and the unit cube, respectively. The general case of multi-linear reference mappings will be subject of a forthcoming paper.

This paper is organised in the following way. Section 2 introduces the basic notation. A general convergence result for nonconforming discretisations of the Stokes problem will be recalled in Section 3. Families of scalar nonconforming finite elements of arbitrary order are defined in Section 4. Section 5 is devoted to consistency, stability, and approximation properties of vector-valued versions of the given nonconforming finite elements together with a discontinuous pressure approximation. The numerical results in Section 6 confirm the theoretical predictions and are compared with known conforming finite element discretisations of the Stokes problem. 
Moreover, the number of degrees of freedom and the number of non-zero matrix entries for conforming and nonconforming discretisations will be discussed.

\section{Notation}

Let $\Omega \subset \mathbb{R}^{d}, d=2,3$, be a domain with polygonal $(d=2)$ or polyhedral $(d=3)$ boundary. We are given a family $\left\{\mathcal{T}_{h}\right\}$ of triangulations of $\Omega$ parametrised by a positive parameter $h$ which tends to 0 . We assume that each triangulation $\mathcal{T}_{h}$ consists of a finite number of (open) quadrilateral or hexahedral cells $K$ such that $\bar{\Omega}=\cup_{K \in \mathcal{T}_{h}} \bar{K}$. Let $h_{K}:=\operatorname{diam}(K)$ and $h:=\max _{K \in \mathcal{T}_{h}} h_{K}$. The triangulations are required to be regular in the sense that the intersection of the closures of two different cells is either empty, a common vertex, a common edge, or a common face.

We restrict ourselves in this paper to the case where all cells $K$ are affine equivalent to the unit square $(-1,+1)^{2}$ and the unit cube $(-1,+1)^{3}$, respectively. The general case of multi-linear reference mappings will be subject of a forthcoming study.

Moreover, we assume that the triangulations are shape regular, i.e., there exists a positive constant $C$ independent of the mesh parameter $h$ such that

$$
\frac{h_{K}}{\varrho_{K}} \leq C \quad \forall K \in \mathcal{T}_{h}, h>0,
$$

where $\varrho_{K}$ denotes the maximum diameter of balls which can be inscribed in $\bar{K}$.

In this paper, the edges in a triangulation of a two-dimensional domain and the faces in a triangulation of a three-dimensional domain will be denoted consistently as sides. Let $\mathcal{S}_{h}$ be the set of all sides, $\mathcal{S}_{h}^{i}$ the set of all inner sides, and $\mathcal{S}_{h}^{b}$ the set of all boundary sides. Moreover, let $|K|$ and $|E|$ denote the $d$-measure of the cell $K$ and $(d-1)$-measure of the side $E$, respectively.

For a domain $D \subset \mathbb{R}^{n}, n=1,2,3$, let $P_{k}(D)$ denote the space of polynomials of degree less than or equal to $k$ while $Q_{k}(D)$ is the space of all polynomials of degree less than or equal to $k$ in each variable.

Furthermore, we use the Sobolev spaces $H^{k}(D)$ and $H_{0}^{k}(D)$ for non-negative integers $k$ and $L^{2}(D):=H^{0}(D)$. The vector-valued versions of these spaces will be denoted by corresponding boldface letters. The norms and semi-norms in the scalar and vector-valued versions of $H^{k}(D)$ are indicated by $\|\cdot\|_{m, D}$ and $|\cdot|_{m, D}$, respectively. The inner product in $L^{2}(D)$, its vector-valued and tensor-valued versions is written as $(\cdot, \cdot)_{D}$. To simplify the notation, the subscript $D$ will be omitted if $D=\Omega$. The space $H^{1, h}(\Omega)$ of piecewise $H^{1}$-functions with respect to $\mathcal{T}_{h}$ is given by

$$
H^{1, h}(\Omega):=\left\{v \in L^{2}(\Omega):\left.v\right|_{K} \in H^{1}(K) \forall K \in \mathcal{T}_{h}\right\} .
$$

We define on this space an analogon of $|\cdot|_{1}$ by

$$
|v|_{1, h}:=\left(\sum_{K \in \mathcal{T}_{h}}|v|_{1, K}^{2}\right)^{1 / 2}, \quad v \in H^{1, h}(\Omega) .
$$

For any side $E \in \mathcal{S}_{h}$, we choose a fixed unit normal vector $\boldsymbol{n}_{E}$. If $E$ is a boundary side then $\boldsymbol{n}_{E}$ coincides with the outer unit normal $\boldsymbol{n}$ on $\partial \Omega$. We define for $v \in H^{1, h}(\Omega)$ its jump $[|v|]_{E}$ across a side $E \in \mathcal{S}_{h}^{i}$ by

$$
[|v|]_{E}:=\left.\left(\left.v\right|_{K}\right)\right|_{E}-\left.\left(\left.v\right|_{\widetilde{K}}\right)\right|_{E}
$$

where $K$ and $\widetilde{K}$ are the two cells which are adjacent to $E$ and $\boldsymbol{n}_{E}$ points into $\widetilde{K}$. Furthermore, let $\nabla_{h} v$ and $\operatorname{div}_{\mathrm{h}} \boldsymbol{v}$ denote the piecewise gradient and piecewise divergence such that

$$
\begin{aligned}
\left.\left(\nabla_{h} v\right)\right|_{K} & =\nabla\left(\left.v\right|_{K}\right), & & v \in H^{1, h}(\Omega), \\
\left.\left(\operatorname{div}_{\mathrm{h}} \boldsymbol{v}\right)\right|_{K} & =\operatorname{div}\left(\left.\boldsymbol{v}\right|_{K}\right), & & \boldsymbol{v} \in \boldsymbol{H}^{1, h}(\Omega),
\end{aligned}
$$


hold true.

For a non-negative integer $i$, let $L_{i}(x)$ denote the $i$-th one-dimensional Legendre polynomial normalised such that $L_{i}(1)=1$ for all $i \geq 0$. Note that in this case

$$
\eta_{i}:=\int_{-1}^{+1}\left(L_{i}(x)\right)^{2} \mathrm{~d} x=\frac{2}{2 i+1}
$$

holds true. Furthermore, we set $L_{i j}:=L_{i j}(x, y):=L_{i}(x) L_{j}(y)$. We have

$$
\int_{-1}^{+1} \int_{-1}^{+1} L_{i j}(x, y) L_{m n}(x, y) \mathrm{d} y \mathrm{~d} x=\eta_{i} \eta_{j} \delta_{i m} \delta_{j n}, \quad i, j, m, n \geq 0
$$

due to the orthogonality of the one-dimensional Legendre polynomials where $\delta_{i m}$ is the Kronecker Delta. For the three-dimensional case, let $L_{i j k}:=L_{i j k}(x, y, z):=L_{i}(x) L_{j}(y) L_{k}(z)$ for non-negative integers $i, j, k$.

In this paper, $C$ will denote a generic positive constant which is independent of the mesh parameter $h$. Note that $C$ may have different values at different places.

\section{General Convergence theory}

In this section, we recall from [6] the general convergence results for nonconforming finite element approximations applied to the Stokes problem (1).

Let the boundary data $\boldsymbol{g} \in \boldsymbol{H}^{1 / 2}(\partial \Omega)$ satisfy $(2), \boldsymbol{V}_{\boldsymbol{g}}:=\left\{\boldsymbol{v} \in \boldsymbol{H}^{1}(\Omega):\left.\boldsymbol{v}\right|_{\partial \Omega}=\boldsymbol{g}\right\}, \boldsymbol{V}_{\mathbf{0}}:=\boldsymbol{H}_{0}^{1}(\Omega)$, and $Q:=\left\{q \in L^{2}(\Omega):(q, 1)=0\right\}$.

A weak formulation of the Stokes problem (1) reads as follows:

Find $(\boldsymbol{u}, p) \in \boldsymbol{V}_{\boldsymbol{g}} \times Q$ such that

$$
(\nabla \boldsymbol{u}, \nabla \boldsymbol{v})-(p, \operatorname{div} \boldsymbol{v})+(q, \operatorname{div} \boldsymbol{u})=(\boldsymbol{f}, \boldsymbol{v}) \quad \forall(\boldsymbol{v}, q) \in \boldsymbol{V}_{\mathbf{0}} \times Q .
$$

It is well-known that due to the Babuška-Brezzi condition

$$
\inf _{q \in Q} \sup _{\boldsymbol{v} \in \boldsymbol{V}_{\mathbf{0}}} \frac{(\operatorname{div} \boldsymbol{v}, q)}{|\boldsymbol{v}|_{1}\|q\|_{0}}>0
$$

there is a unique weak solution of (4), see [9].

Let $\boldsymbol{V}_{\boldsymbol{g} h} \approx \boldsymbol{V}_{\boldsymbol{g}}$ (in particular $\boldsymbol{V}_{\mathbf{0} h} \approx \boldsymbol{V}_{\mathbf{0}}$ ) be a nonconforming finite element space for approximating the velocity where we assume that $\boldsymbol{V}_{\mathbf{0} h}=\boldsymbol{V}_{\boldsymbol{g} h}$ for $\boldsymbol{g}=\mathbf{0}$. Furthermore, let $Q_{h} \subset Q$ be a finite element space for approximating the pressure.

The discrete problem reads as follows:

Find $\left(\boldsymbol{u}_{h}, p_{h}\right) \in \boldsymbol{V}_{\boldsymbol{g} h} \times Q_{h}$ such that

$$
\left(\nabla_{h} \boldsymbol{u}_{h}, \nabla_{h} \boldsymbol{v}_{h}\right)-\left(p_{h}, \operatorname{div}_{\mathbf{h}} \boldsymbol{v}_{h}\right)+\left(q_{h}, \operatorname{div}_{\mathbf{h}} \boldsymbol{u}_{h}\right)=\left(\boldsymbol{f}, \boldsymbol{v}_{h}\right) \quad \forall\left(\boldsymbol{v}_{h}, q_{h}\right) \in \boldsymbol{V}_{\mathbf{0} h} \times Q_{h} .
$$

We fix the order $r \geq 1$ and assume that the following conditions are fulfilled. 
(H1) Consistency:

We have

$$
\int_{E} q\left[\left|\boldsymbol{v}_{h}\right|\right]_{E} \mathrm{~d} \gamma=\mathbf{0} \quad \forall q \in P_{r-1}(E), \boldsymbol{v}_{h} \in \boldsymbol{V}_{\boldsymbol{g} h},
$$

for any inner side $E \in \mathcal{S}_{h}^{i}$ and

$$
\int_{E} q \boldsymbol{v}_{h} \mathrm{~d} \gamma=\int_{E} q \boldsymbol{g} \mathrm{d} \gamma \quad \forall q \in P_{r-1}(E), \boldsymbol{v}_{h} \in \boldsymbol{V}_{\boldsymbol{g} h},
$$

for any boundary side $E \in \mathcal{S}_{h}^{b}$.

(H2) Stability:

The discrete version of (5) is valid uniformly in $h$, i.e.,

$$
\exists \beta>0 \forall h: \quad \inf _{q_{h} \in Q_{h}} \sup _{\boldsymbol{v}_{h} \in \boldsymbol{V}_{\mathbf{o}}} \frac{\left(\operatorname{div}_{\mathrm{h}} \boldsymbol{v}_{h}, q_{h}\right)}{\left|\boldsymbol{v}_{h}\right|_{1, h}\left\|q_{h}\right\|_{0}} \geq \beta .
$$

\section{(H3) Approximation properties:}

There are interpolation operators $\boldsymbol{i}_{h}: \boldsymbol{V}_{\boldsymbol{g}} \cap \boldsymbol{H}^{r+1}(\Omega) \rightarrow \boldsymbol{V}_{\boldsymbol{g} h}$ and $j_{h}: Q \cap H^{r}(\Omega) \rightarrow Q_{h}$ such that

$$
\begin{aligned}
\left|\boldsymbol{u}-\boldsymbol{i}_{h} \boldsymbol{u}\right|_{1, h} & \leq C h^{r}|\boldsymbol{u}|_{r+1} & & \forall \boldsymbol{u} \in \boldsymbol{V}_{\boldsymbol{g}} \cap \boldsymbol{H}^{r+1}(\Omega), \\
\left\|p-j_{h} p\right\|_{0} & \leq C h^{r}|p|_{r} & & \forall p \in Q \cap H^{r}(\Omega) .
\end{aligned}
$$

Note that (H1) implies that $|\cdot|_{1, h}$ is a norm on $\boldsymbol{V}_{\mathbf{0} h}$. Our assumptions guarantee the existence and uniqueness of a solution $\left(\boldsymbol{u}_{h}, p_{h}\right) \in \boldsymbol{V}_{\boldsymbol{g} h} \times Q_{h}$ of the discrete problem (6). Moreover, the error estimate

$$
\left|\boldsymbol{u}-\boldsymbol{u}_{h}\right|_{1, h}+\left\|p-p_{h}\right\|_{0} \leq C h^{r}\left(|\boldsymbol{u}|_{r+1}+|p|_{r}\right)
$$

holds true $[6,9]$.

\section{SCALAR FAMilies of NONCONFORMing Finite ELEMENTS}

We will define in this section four families of scalar nonconforming finite elements, three for the 2D case and one for the 3D case. First, we will define the finite elements on the corresponding reference cells. The actually used finite elements are obtained by using suitable reference mappings.

\subsection{Finite elements on quadrilaterals}

We will start with the two-dimensional families. Let $\widehat{K}=(-1,+1)^{2}$ denote the reference square. Its sides are denoted counter-clockwise by $A, B, C$, and $D$, as shown in Figure 1 . We define for $j \geq 0$ the following nodal functionals

$$
\begin{array}{ll}
\widehat{N}_{A, j}(v):=\eta_{j}^{-1} \int_{-1}^{+1} v(x,-1) L_{j}(x) \mathrm{d} x, & \widehat{N}_{B, j}(v):=\eta_{j}^{-1} \int_{-1}^{+1} v(+1, y) L_{j}(y) \mathrm{d} y, \\
\widehat{N}_{C, j}(v):=\eta_{j}^{-1} \int_{-1}^{+1} v(x,+1) L_{j}(x) \mathrm{d} x, & \widehat{N}_{D, j}(v):=\eta_{j}^{-1} \int_{-1}^{+1} v(-1, y) L_{j}(y) \mathrm{d} y .
\end{array}
$$




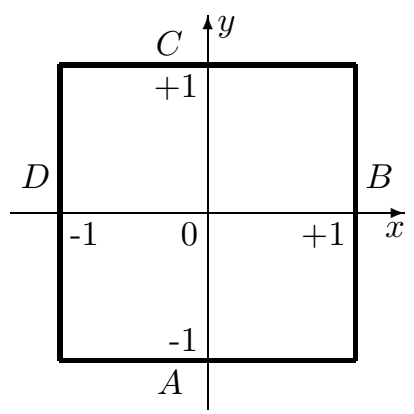

Figure 1. Side labels on the reference cell $\widehat{K}=(-1,+1)^{2}$.

Furthermore, we introduce for $i, j \geq 0$ the nodal functionals

$$
\widehat{N}_{i j}(v):=\left(\eta_{i} \eta_{j}\right)^{-1} \int_{-1}^{+1} \int_{-1}^{+1} v(x, y) L_{i j}(x, y) \mathrm{d} x \mathrm{~d} y .
$$

We define the set $\widehat{\mathcal{N}}_{r}$ of nodal functionals by

$$
\widehat{\mathcal{N}}_{r}:=\left\{\widehat{N}_{A, j}, \widehat{N}_{B, j}, \widehat{N}_{C, j}, \widehat{N}_{D, j}, j=0, \ldots, r-1\right\} \cup\left\{\widehat{N}_{i j}: 0 \leq i+j \leq r-2\right\} .
$$

Note that the set $\widehat{\mathcal{N}}_{r}$ contains $r(r+7) / 2$ nodal functionals.

Now we are looking for polynomials spaces $\widehat{V}_{r}$ of dimension $r(r+7) / 2$ which on the one hand contain $P_{r}(\widehat{K})$ and which on the other hand are unisolvent with respect to $\widehat{\mathcal{N}}_{r}$. We will give three possible choices.

A first choice was already given in Example 5 in [11]. We set

$$
\widehat{V}_{r}^{1}:=P_{r}(\widehat{K}) \oplus \widehat{R}_{r} \oplus \widehat{S}_{r}
$$

with

$$
\widehat{S}_{r}:=\operatorname{span}\left\{L_{i i}, L_{i+1, i}, L_{i, i+1}, L_{i+2, i}-L_{i, i+2}: r / 2<i \leq r-1\right\}
$$

and

$$
\widehat{R}_{r}:= \begin{cases}\operatorname{span}\left\{L_{i+1, i}, L_{i, i+1}, L_{i+2, i}-L_{i, i+2},: i=r / 2\right\}, & r \text { even, } \\ \operatorname{span}\left\{L_{i+2, i}-L_{i, i+2}: i=(r-1) / 2\right\}, & r \text { odd. }\end{cases}
$$

The space $\widehat{V}_{r}^{1}$ is the output of Algorithm 1 in [11] which ensures the unisolvence and that $\operatorname{dim} \widehat{V}_{r}^{1}=r(r+7) / 2$, see [11], Lemma 1. Note that $k$ in [11] corresponds to $r-1$ in our paper.

We propose two new choices $\widehat{V}_{r}^{2}$ and $\widehat{V}_{r}^{3}$ for $\widehat{V}_{r}$ which are given by

$$
\begin{aligned}
\widehat{V}_{r}^{2}:=P_{r}(\widehat{K}) & \oplus \operatorname{span}\left\{L_{i+2, r-1-i}-L_{i, r+1-i}, i=0, \ldots, r-1\right\} \\
& \oplus \operatorname{span}\left\{L_{i+2, r-i}-L_{i, r+2-i}, i=1, \ldots, r-1\right\}
\end{aligned}
$$



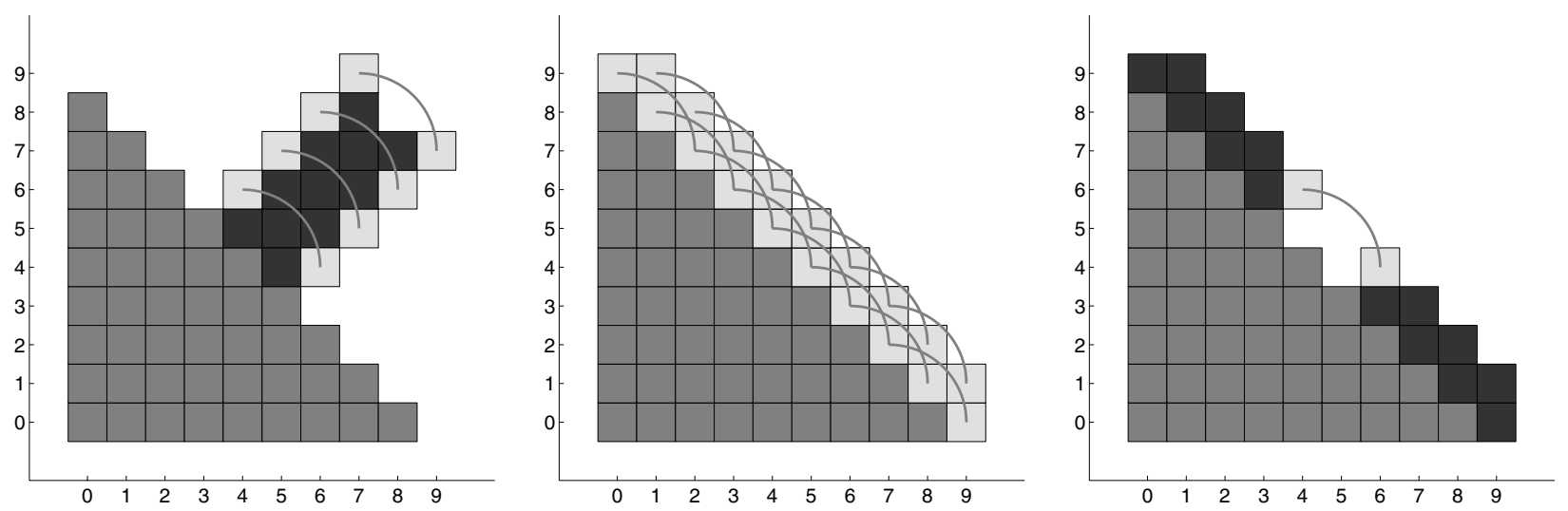

FIgURE 2. Illustration of the function spaces $\widehat{V}_{r}^{\nu}$ for $r=8, \nu=1,2,3$, from left bottom right.

and

$$
\begin{aligned}
\widehat{V}_{r}^{3}:=P_{r}(\widehat{K}) & \oplus \operatorname{span}\left\{L_{i, r+1-i}, L_{r+1-i, i}, i=0, \ldots,\lfloor r / 2\rfloor-1\right\} \\
& \oplus \operatorname{span}\left\{L_{i, r+2-i}, L_{r+2-i, i}, i=1, \ldots,\lfloor(r+1) / 2\rfloor-1\right\} \\
& \oplus \operatorname{span}\left\{L_{\lfloor r / 2\rfloor+2,\lfloor r / 2\rfloor}-L_{\lfloor r / 2\rfloor,\lfloor r / 2\rfloor+2}\right\}
\end{aligned}
$$

where $\lfloor x\rfloor$ is the largest integer which is less than or equal to $x$. One easily checks that $\operatorname{dim} \widehat{V}_{r}^{2}=\operatorname{dim} \widehat{V}_{r}^{3}=$ $r(r+7) / 2$. The proof of the unisolvence of $\widehat{\mathcal{N}}_{r}$ on $\widehat{V}_{r}^{2}$ and $\widehat{V}_{r}^{3}$ will be given in Lemmas 4.1 and 4.2 , later in this section.

We have in the case $r=1$ that

$$
\widehat{V}_{1}^{1}=\widehat{V}_{1}^{2}=\widehat{V}_{1}^{3}=\operatorname{span}\left\{L_{00}, L_{10}, L_{01}, L_{20}-L_{02}\right\}=\operatorname{span}\left\{1, x, y, x^{2}-y^{2}\right\}
$$

hence, the rotated bilinear element by Rannacher and Turek [19] is recovered. Note that the polynomial spaces $\widehat{V}_{r}^{1}, \widehat{V}_{r}^{2}$, and $\widehat{V}_{r}^{3}$ differ for $r \geq 2$. Indeed, we obtain for $r=2$ the spaces

$$
\begin{array}{ll}
\widehat{V}_{2}^{1}=P_{2}(\widehat{K}) \oplus \operatorname{span}\left\{L_{21}, L_{12}, L_{31}-L_{13}\right\} & =P_{2}(\widehat{K}) \oplus \operatorname{span}\left\{x^{2} y, x y^{2}, x^{3} y-x y^{3}\right\} \\
\widehat{V}_{2}^{2}=P_{2}(\widehat{K}) \oplus \operatorname{span}\left\{L_{21}-L_{03}, L_{30}-L_{12}, L_{31}-L_{13}\right\} & =P_{2}(\widehat{K}) \oplus \operatorname{span}\left\{x\left(x^{2}-y^{2}\right), y\left(x^{2}-y^{2}\right), x^{3} y-x y^{3}\right\} \\
\widehat{V}_{2}^{3}=P_{2}(\widehat{K}) \oplus \operatorname{span}\left\{L_{30}, L_{03}, L_{31}-L_{13}\right\} & =P_{2}(\widehat{K}) \oplus \operatorname{span}\left\{x^{3}, y^{3}, x^{3} y-x y^{3}\right\}
\end{array}
$$

Since the families $\widehat{V}_{r}^{1}, \widehat{V}_{r}^{2}$, and $\widehat{V}_{r}^{3}$ are unisolvent with respect to the set $\widehat{\mathcal{N}}_{r}$ of nodal functionals, one has the freedom of choice. The families differ in some properties. On the one hand, the space $\widehat{V}_{r}^{2}$ contains for $r \geq 2$ more differences of products of Legendre polynomials than $\widehat{V}_{r}^{1}$ and $\widehat{V}_{r}^{3}$. On the other hand, $\widehat{V}_{r}^{2}$ and $\widehat{V}_{r}^{3}$ are a subsets of $P_{r+2}(\widehat{K})$ while $\widehat{V}_{r}^{1}$ contains contributions up to $P_{2 r}(\widehat{K})$. An illustration of the obtained spaces for $r=8$ is given in Figure 2 where the grey boxes indicate the space $P_{r}(\widehat{K})$, the dark boxes stand for further functions of form $L_{i}(x) L_{j}(y)$, and if a pair of bright boxes is connected by an arc then only the difference of the involved functions belongs to the space $\widehat{V}_{r}$.

We continue by proving the unisolvence of $\widehat{\mathcal{N}}_{r}$ with respect to the spaces $\widehat{V}_{r}^{2}$ and $\widehat{V}_{r}^{3}$.

Lemma 4.1. The set $\widehat{\mathcal{N}}_{r}$ of nodal functionals is unisolvent on $\widehat{V}_{r}^{2}$. 
Proof. We will prove that $v \equiv 0$ follows from $v \in \widehat{V}_{r}^{2}$ and $\widehat{N}(v)=0$ for all $\widehat{N} \in \widehat{\mathcal{N}}_{r}$.

We get from (11) the representation

$$
v=\sum_{0 \leq i+j \leq r} \alpha_{i j} L_{i j}+\sum_{i=0}^{r-1} \beta_{i+1}\left(L_{i+2, r-1-i}-L_{i, r+1-i}\right)+\sum_{i=1}^{r-1} \gamma_{i+1}\left(L_{i+2, r-i}-L_{i, r+2-i}\right)
$$

since

$$
P_{r}(\widehat{K})=\operatorname{span}\left\{L_{i j}: 0 \leq i+j \leq r\right\} .
$$

By using $\widehat{N}_{m n}(v)=0$ for $0 \leq m+n \leq r-2$ and the orthogonality (3), we obtain

$$
\alpha_{m n}=0, \quad 0 \leq m+n \leq r-2 .
$$

Hence, the representation of $v$ simplifies to

$$
v=\sum_{i=0}^{r-1} \alpha_{i, r-1-i} L_{i, r-1-i}+\sum_{i=0}^{r} \alpha_{i, r-i} L_{i, r-i}+\sum_{i=0}^{r-1} \beta_{i+1}\left(L_{i+2, r-1-i}-L_{i, r+1-i}\right)+\sum_{i=1}^{r-1} \gamma_{i+1}\left(L_{i+2, r-i}-L_{i, r+2-i}\right) .
$$

Exploiting the fact that $L_{s}(1)=1$ for all $s \geq 0$, we get from $\widehat{N}_{B, n}(v)=0, n=0, \ldots, r-1$,

$$
0=\left(\alpha_{r-1-n, n}+\alpha_{r-n, n}\right)+\left(\beta_{r-n}-\beta_{r+2-n}\right)+\left(\gamma_{r+1-n}-\gamma_{r+3-n}\right), \quad n=0, \ldots, r-1 .
$$

Note that we set $\beta_{i}=0$ for $i<1$ or $i>r$ and $\gamma_{i}=0$ for $i<2$ or $i>r$. Incorporating that $L_{s}(-1)=(-1)^{s}$ for all $s \geq 0$, we obtain by using $\widehat{N}_{D, n}(v)=0, n=0, \ldots, r-1$,

$$
0=\left(\alpha_{r-1-n, n}-\alpha_{r-n, n}\right)+\left(\beta_{r-n}-\beta_{r+2-n}\right)-\left(\gamma_{r+1-n}-\gamma_{r+3-n}\right), \quad n=0, \ldots, r-1 .
$$

Taking the sum and difference of (13) and (14), we get

$$
0=\alpha_{r-1-n, n}+\beta_{r-n}-\beta_{r+2-n}, \quad n=0, \ldots, r-1,
$$

and

$$
0=\alpha_{r-n, n}+\gamma_{r+1-n}-\gamma_{r+3-n}, \quad n=0, \ldots, r-1,
$$

respectively.

By using $\widehat{N}_{C, m}(v)=0$ and $\widehat{N}_{A, m}(v)=0$ for $m=0, \ldots, r-1$, we obtain

$$
0=\left(\alpha_{m, r-1-m}+\alpha_{m, r-m}\right)+\left(\beta_{m-1}-\beta_{m+1}\right)+\left(\gamma_{m-1}-\gamma_{m+1}\right), \quad m=0, \ldots, r-1,
$$

and

$$
0=\left(\alpha_{m, r-1-m}-\alpha_{m, r-m}\right)+\left(\beta_{m-1}-\beta_{m+1}\right)-\left(\gamma_{m-1}-\gamma_{m+1}\right), \quad m=0, \ldots, r-1 .
$$

The sum and difference of (17) and (18) result in

$$
0=\alpha_{m, r-1-m}+\beta_{m-1}-\beta_{m+1}, \quad m=0, \ldots, r-1,
$$


and

$$
0=\alpha_{m, r-m}+\gamma_{m-1}-\gamma_{m+1}, \quad m=0, \ldots, r-1
$$

respectively.

The difference of (15) with $n$ and (19) with $m=r-1-n$ gives

$$
-\beta_{r-2-n}+2 \beta_{r-n}-\beta_{r+2-n}=0, \quad n=0, \ldots, r-1 .
$$

This is a system of linear equations for $\beta_{i}, i=1, \ldots, r$, where $\beta_{0}=\beta_{-1}=\beta_{r+1}=\beta_{r+2}=0$ are regarded as known values. A closer look at the system (21) shows that it decomposes into two independent sub-systems of homogeneous linear equations: one with all variables having odd indices and one with all variables having even indices. The system matrix in both cases is tridiagonal with 2 on the diagonal and -1 on the sub-diagonals. Hence, the matrix is as M-matrix of course regular. This yields that both sub-systems have only the trivial solution. This gives $\beta_{n}=0$ for all $n=1, \ldots, r$. Putting this into (15), we get $\alpha_{r-1-n, n}=0$ for $n=0, \ldots, r-1$.

Using the difference of (16) with $n$ and (20) with $m=r-n$ for $n=1, \ldots, r-1$, we end up with a system of type (21), but for $\gamma_{n}, n=2, \ldots, r$. Applying the same arguments as before, we get $\gamma_{n}=0$ for $n=2, \ldots, r$, and, by (20), $\alpha_{r-n, n}=0$ for $n=1, \ldots, r-1$. It remains to show that the two not yet considered coefficients $\alpha_{r, 0}$ and $\alpha_{0, r}$ will also vanish. This follows immediately if we consider (16) for $n=0$ and (20) for $m=0$.

Hence, we have shown that $v \in \widehat{V}_{r}^{2}$ and $\widehat{N}(v)=0$ for all $\widehat{N} \in \widehat{\mathcal{N}}_{r}$ implies $v \equiv 0$. This means, the set $\widehat{\mathcal{N}}_{r}$ is unisolvent on $\widehat{V}_{r}^{2}$.

Lemma 4.2. The set $\widehat{\mathcal{N}}_{r}$ of nodal functionals is unisolvent on $\widehat{V}_{r}^{3}$.

Proof. Now we prove that $v \in \widehat{V}_{r}^{3}$ and $\widehat{N}(v)=0$ for all $\widehat{N} \in \widehat{\mathcal{N}}_{r}$ give $v \equiv 0$. We will restrict ourselves to the case where $r$ is even since the proof for odd $r$ goes in a similar way.

We get from (12) that $v$ can be written as

$$
\begin{aligned}
v= & \sum_{0 \leq i+j \leq r} \alpha_{i j} L_{i j}+\delta\left(L_{r / 2+2, r / 2}-L_{r / 2, r / 2+2}\right) \\
& +\sum_{i=0}^{r / 2-1} \beta_{i} L_{i, r+1-i}+\sum_{i=r / 2+2}^{r+1} \beta_{i} L_{i, r+1-i}+\sum_{i=1}^{r / 2-1} \gamma_{i} L_{i, r+2-i}+\sum_{i=r / 2+3}^{r+1} \gamma_{i} L_{i, r+2-i} .
\end{aligned}
$$

Exploiting $\widehat{N}_{m n}(v)=0$ for $0 \leq m+n \leq r-2$ and the orthogonality of the Legendre polynomials, we obtain

$$
\alpha_{m n}=0, \quad 0 \leq m+n \leq r-2 .
$$

Hence, $v$ can be written as

$$
\begin{aligned}
v= & \sum_{i=0}^{r-1} \alpha_{i, r-1-i} L_{i, r-1-i}+\sum_{i=0}^{r} \alpha_{i, r-i} L_{i, r-i}+\delta\left(L_{r / 2+2, r / 2}-L_{r / 2, r / 2+2}\right) \\
& +\sum_{i=0}^{r / 2-1} \beta_{i} L_{i, r+1-i}+\sum_{i=r / 2+2}^{r+1} \beta_{i} L_{i, r+1-i}+\sum_{i=1}^{r / 2-1} \gamma_{i} L_{i, r+2-i}+\sum_{i=r / 2+3}^{r+1} \gamma_{i} L_{i, r+2-i}
\end{aligned}
$$

Using $\widehat{N}_{A, r / 2+1}(v)=0$ and $\widehat{N}_{C, r / 2+1}(v)=0$, we get

$$
0=\alpha_{r / 2+1, r / 2-1}+\alpha_{r / 2+1, r / 2-2}, \quad 0=\alpha_{r / 2+1, r / 2-1}-\alpha_{r / 2+1, r / 2-2}
$$


which gives

$$
\alpha_{r / 2+1, r / 2-1}=\alpha_{r / 2+1, r / 2-2}=0 .
$$

Analogously, the use of $\widehat{N}_{B, r / 2+1}(v)=\widehat{N}_{D, r / 2+1}(v)=0$ results in

$$
\alpha_{r / 2-1, r / 2+1}=\alpha_{r / 2-2, r / 2+1}=0 .
$$

The application of $\widehat{N}_{A, r / 2}(v)=\widehat{N}_{B, r / 2}(v)=\widehat{N}_{C, r / 2}(v)=\widehat{N}_{D, r / 2}(v)=0$ yields

$$
\begin{array}{ll}
0=\alpha_{r / 2, r / 2}+\alpha_{r / 2, r / 2-1}-\delta, & 0=\alpha_{r / 2, r / 2}+\alpha_{r / 2-1, r / 2}+\delta \\
0=\alpha_{r / 2, r / 2}-\alpha_{r / 2, r / 2-1}-\delta, & 0=\alpha_{r / 2, r / 2}-\alpha_{r / 2-1, r / 2}+\delta .
\end{array}
$$

We obtain from this that

$$
\alpha_{r / 2, r / 2-1}=\alpha_{r / 2-1, r / 2}=\alpha_{r / 2, r / 2}=\delta=0 .
$$

By using $\widehat{N}_{B, r / 2-1}(v)=\widehat{N}_{D, r / 2-1}(v)=0$, we get

$$
0=\beta_{r / 2+2}+\gamma_{r / 2+3}, \quad 0=\beta_{r / 2+2}-\gamma_{r / 2+3},
$$

which shows that

$$
\beta_{r / 2+2}=\gamma_{r / 2+3}=0 .
$$

Applying $\widehat{N}_{A, r / 2-1}(v)=\widehat{N}_{C, r / 2-1}(v)=0$ gives in the same way,

$$
\beta_{r / 2-1}=\gamma_{r / 2-1}=0 .
$$

An alternating use of $\widehat{N}_{A, r / 2+m}(v)=\widehat{N}_{A, r / 2+m}(v)=0$ and $\widehat{N}_{B, r / 2-m}(v)=\widehat{N}_{D, r / 2-m}(v)=0$ for $m=$ $2, \ldots, r / 2-1$ gives

$$
\alpha_{r / 2+m, r / 2-1-m}=\alpha_{r / 2+m, r / 2-m}=0, \quad m=2, \ldots, r / 2-1,
$$

and

$$
\beta_{r / 2+1+m}=\gamma_{r / 2+2+m}=0, \quad m=2, \ldots, r / 2-1,
$$

respectively. By similar arguments and by exploiting $\widehat{N}_{B, r / 2+m}(v)=\widehat{N}_{D, r / 2+m}(v)=0$ and $\widehat{N}_{A, r / 2-m}(v)=$ $\widehat{N}_{C, r / 2-m}(v)=0$ for $m=2, \ldots, r / 2-1$, we get

$$
\alpha_{r / 2-1-m, r / 2+m}=\alpha_{r / 2-m, r / 2+m}=0, \quad m=2, \ldots, r / 2-1,
$$

and

$$
\beta_{r / 2-m}=\gamma_{r / 2-m}=0, \quad m=2, \ldots, r / 2-1 .
$$

Finally, the use of $\widehat{N}_{B, 0}(v)=\widehat{N}_{D, 0}(v)=0$ yields

$$
0=\alpha_{r, 0}+\beta_{r+1}, \quad 0=\alpha_{r, 0}-\beta_{r+1}
$$

showing that

$$
\alpha_{r, 0}=\beta_{r+1}=0
$$


holds true. Analogously, we get

$$
\alpha_{0, r}=\beta_{0}=0
$$

from $\widehat{N}_{A, 0}(v)=\widehat{N}_{C, 0}(v)=0$.

To summarise, all coefficients in the representation (22) of $v$ vanish. This means, we have shown that $v \in \widehat{V}_{r}^{3}$ and $\widehat{N}(v)=0$ for all $\widehat{N} \in \widehat{\mathcal{N}}_{r}$ gives $v \equiv 0$. Hence, $\widehat{\mathcal{N}}_{r}$ is unisolvent on $\widehat{V}_{r}^{3}$.

\subsection{Finite elements on hexahedra}

Now we consider the three-dimensional case where we will apply again results from [11]. To this end, we define on the reference cell $\widehat{K}=(-1,+1)^{3}$ for non-negative integers $i, j$ the following nodal functionals

$$
\begin{aligned}
& \widehat{N}_{U, i j}(v):=\left(\eta_{i} \eta_{j}\right)^{-1} \int_{-1}^{+1} \int_{-1}^{+1} v(x, y,+1) L_{i}(x) L_{j}(y) \mathrm{d} x \mathrm{~d} y, \\
& \widehat{N}_{D, i j}(v):=\left(\eta_{i} \eta_{j}\right)^{-1} \int_{-1}^{+1} \int_{-1}^{+1} v(x, y,-1) L_{i}(x) L_{j}(y) \mathrm{d} x \mathrm{~d} y, \\
& \widehat{N}_{L, i j}(v):=\left(\eta_{i} \eta_{j}\right)^{-1} \int_{-1}^{+1} \int_{-1}^{+1} v(-1, y, z) L_{i}(y) L_{j}(z) \mathrm{d} y \mathrm{~d} z, \\
& \widehat{N}_{R, i j}(v):=\left(\eta_{i} \eta_{j}\right)^{-1} \int_{-1}^{+1} \int_{-1}^{+1} v(+1, y, z) L_{i}(y) L_{j}(z) \mathrm{d} y \mathrm{~d} z, \\
& \widehat{N}_{F, i j}(v):=\left(\eta_{i} \eta_{j}\right)^{-1} \int_{-1}^{+1} \int_{-1}^{+1} v(x,-1, z) L_{i}(x) L_{j}(z) \mathrm{d} x \mathrm{~d} z, \\
& \widehat{N}_{B, i j}(v):=\left(\eta_{i} \eta_{j}\right)^{-1} \int_{-1}^{+1} \int_{-1}^{+1} v(x,+1, z) L_{i}(x) L_{j}(z) \mathrm{d} x \mathrm{~d} z .
\end{aligned}
$$

Furthermore, we introduce for $i, j, k \geq 0$ the nodal functionals

$$
\widehat{N}_{i j k}(v):=\left(\eta_{i} \eta_{j} \eta_{k}\right)^{-1} \int_{-1}^{+1} \int_{-1}^{+1} \int_{-1}^{+1} v(x, y, z) L_{i j k}(x, y, z) \mathrm{d} x \mathrm{~d} y \mathrm{~d} z .
$$

The set $\widehat{\mathcal{N}}_{r}$ of nodal functionals is given by

$$
\begin{gathered}
\widehat{\mathcal{N}}_{r}:=\left\{\widehat{N}_{U, i j}, \widehat{N}_{D, i j}, \widehat{N}_{L, i j}, \widehat{N}_{R, i j}, \widehat{N}_{F, i j}, \widehat{N}_{B, i j}: 0 \leq i+j \leq r-1\right\} \\
\cup\left\{\widehat{N}_{i j k}: 0 \leq i+j+k \leq r-2\right\}
\end{gathered}
$$

and contains $r(r+1)(r+17) / 6$ elements. One can construct by using Algorithm 2 from [11] a polynomial space $\widehat{V}_{r}$ of dimension $r(r+1)(r+17) / 6$ which on the one hand contains $P_{r}(\widehat{K})$ and which on the other hand is unisolvent with respect to the nodal functionals from $\widehat{\mathcal{N}}_{r}$, see [11], Lemma 2. Example 10 in [11] presents the 
obtained space $\widehat{V}_{r}$ which is given as

$$
\widehat{V}_{r}:=P_{r-2}(\widehat{K}) \oplus \bigoplus_{i=1}^{3} \widehat{A}_{i} \oplus \bigoplus_{i=1}^{3} \widehat{B}_{i} \oplus \widehat{C}
$$

with

$$
\begin{aligned}
& \widehat{A}_{1}:=\operatorname{span}\left\{L_{i, j, k}, L_{i+1, j, k}: i>\max (j, k), i+j+k=r-1\right\}, \\
& \widehat{A}_{2}:=\operatorname{span}\left\{L_{i, j, k}, L_{i, j+1, k}: j>\max (i, k), i+j+k=r-1\right\}, \\
& \widehat{A}_{3}:=\operatorname{span}\left\{L_{i, j, k}, L_{i, j, k+1}: k>\max (i, j), i+j+k=r-1\right\},
\end{aligned}
$$

and

$$
\begin{aligned}
& \widehat{B}_{1}:=\operatorname{span}\left\{L_{j i i}, L_{j, i+1, i}, L_{j, i, i+1}, L_{j, i, i+2}-L_{j, i+2, i}: i>j, r-1 \leq 2 i+j \leq 2(r-1)-j\right\}, \\
& \widehat{B}_{2}:=\operatorname{span}\left\{L_{i j i}, L_{i+1, j, i}, L_{i, j, i+1}, L_{i, j, i+2}-L_{i+2, j, i}: i>j, r-1 \leq 2 i+j \leq 2(r-1)-j\right\}, \\
& \widehat{B}_{3}:=\operatorname{span}\left\{L_{i i j}, L_{i+1, i, j}, L_{i, i+1, j}, L_{i, i+2, j}-L_{i+2, i, j}: i>j, r-1 \leq 2 i+j \leq 2(r-1)-j\right\} .
\end{aligned}
$$

Furthermore, we set

$$
\begin{aligned}
\widehat{C}:= & \operatorname{span}\left\{L_{i i i}, L_{i+1, i, i}, L_{i, i+1, i}, L_{i, i, i+1}:(r-1) / 3 \leq i \leq(r-1) / 2\right\} \\
& \oplus \operatorname{span}\left\{\alpha L_{i+2, i, i}+\beta L_{i, i+2, i}+\gamma L_{i, i, i+2} \text { with } \alpha+\beta+\gamma=0:(r-1) / 3 \leq i \leq(r-1) / 2\right\} .
\end{aligned}
$$

It is also shown in [11] that $P_{r}(\widehat{K}) \subset \widehat{V}_{r}$ holds true. Note that $l$ in [11] corresponds to $r-1$ in our paper.

One gets in the case $r=1$ the well-known element by Rannacher and Turek [19]. We have for $r=2$ the spaces

$$
\begin{array}{ll}
\widehat{A}_{1}=\operatorname{span}\left\{L_{100}, L_{200}\right\}, & \widehat{B}_{1}=\operatorname{span}\left\{L_{011}, L_{021}, L_{012}, L_{031}-L_{013}\right\}, \\
\widehat{A}_{2}=\operatorname{span}\left\{L_{010}, L_{020}\right\}, & \widehat{B}_{2}=\operatorname{span}\left\{L_{101}, L_{201}, L_{102}, L_{301}-L_{103}\right\}, \quad \widehat{C}=\emptyset . \\
\widehat{A}_{3}=\operatorname{span}\left\{L_{001}, L_{002}\right\}, & \widehat{B}_{3}=\operatorname{span}\left\{L_{110}, L_{210}, L_{120}, L_{310}-L_{130}\right\},
\end{array}
$$

Hence, the representation

$$
\widehat{V}_{2}=P_{2}(\widehat{K}) \oplus \operatorname{span}\left\{L_{012}, L_{021}, L_{102}, L_{201}, L_{201}, L_{210}\right\} \oplus \operatorname{span}\left\{L_{013}-L_{031}, L_{103}-L_{301}, L_{130}-L_{310}\right\}
$$

is obtained.

\subsection{Finite element spaces}

Using the finite elements $\left(\widehat{K}, \widehat{V}_{r}, \widehat{\mathcal{N}}_{r}\right)$ on the reference cell $\widehat{K}$, we can define on each cell $K$ the finite elements $\left(K, V_{r}(K), \mathcal{N}_{r}(K)\right)$. The function space $V_{r}(K)$ is defined as follows

$$
V_{r}(K):=\left\{v=\hat{v} \circ F_{K}^{-1}: \hat{v} \in \widehat{V}_{r}\right\}
$$

where $F_{K}: \widehat{K} \rightarrow K$ denotes the invertible and affine reference mapping. 
The global scalar nonconforming finite element space $V_{g h}$ is defined by

$$
\begin{aligned}
V_{g h}:= & \left\{\begin{array}{l}
v \in H^{1, h}(\Omega):\left.v\right|_{K} \in V_{r}(K) \quad \forall K \in \mathcal{T}_{h}, \\
\left.\quad \int_{E} q[|v|]_{E} \mathrm{~d} \gamma=0 \forall E \in \mathcal{S}_{h}^{i} \forall q \in P_{r-1}(E), \int_{E} q(v-g) \mathrm{d} \gamma=0 \quad \forall E \in \mathcal{S}_{h}^{b} \forall q \in P_{r-1}(E)\right\} .
\end{array}\right.
\end{aligned}
$$

Note that this definition is common for the two-dimensional and the three-dimensional case.

\section{Consistency, Stability And Approximation PROPERTies}

We will show in this section that the hypotheses (H1), (H2), and (H3) are fulfilled for each family of nonconforming finite elements together with a discontinuous piecewise polynomial pressure. We fix the order parameter $r$. The $d$ velocity components will be approximated by $V_{g h}$ while the pressure is approximated by the space $P_{r-1}^{\text {disc }}$ of discontinuous, piecewise $P_{r-1}$-elements.

The consistency (H1) follows immediately from the vector-valued generalisation of the definition (24) of the space $V_{g h}$.

In order to prove the approximation property (H3), we introduce first local (scalar) interpolation operators

$$
\widehat{I}_{r}: H^{1}(\widehat{K}) \rightarrow \widehat{V}_{r}, \quad \widehat{J}_{r}: L^{2}(\widehat{K}) \rightarrow P_{r-1}(\widehat{K})
$$

on the reference cell $\widehat{K}$. Let $\widehat{J}_{r}$ be the $L^{2}(\widehat{K})$-projection onto $P_{r-1}(\widehat{K})$, i.e.,

$$
\left(\widehat{J_{r}} \hat{p}, \hat{q}\right)_{\widehat{K}}=(\hat{p}, \hat{q})_{\widehat{K}} \quad \forall \hat{q} \in P_{r-1}(\widehat{K}) .
$$

Using $n=\operatorname{dim} \widehat{V}_{r}$ and the nodal functionals from $\widehat{\mathcal{N}}_{r}=\left\{\widehat{N}_{i}: i=1, \ldots, n\right\}$, we define

$$
\widehat{I}_{r} \hat{v}:=\sum_{i=1}^{n} \widehat{N}_{i}(\hat{v}) \hat{\varphi}_{i}
$$

where $\left\{\hat{\varphi}_{i}: i=1, \ldots, n\right\}$ is the dual basis to $\widehat{\mathcal{N}}_{r}$, i.e., $\widehat{N}_{i}\left(\hat{\varphi}_{j}\right)=\delta_{i j}, i, j=1, \ldots, n$.

The interpolation operators

$$
I_{r}^{K}: H^{1}(K) \rightarrow V_{r}(K), \quad J_{r}^{K}: L^{2}(K) \rightarrow P_{r-1}(K)
$$

are defined as

$$
I_{r}^{K} v:=\left(\widehat{I}_{r}\left(v \circ F_{K}\right)\right) \circ F_{K}^{-1}, \quad J_{r}^{K} q:=\left(\widehat{J}_{r}\left(q \circ F_{K}\right)\right) \circ F_{K}^{-1} .
$$

Now we can give estimates for the local interpolation operators.

Lemma 5.1. The local interpolation operators $I_{r}^{K}: H^{1}(K) \rightarrow V_{r}(K)$ and $J_{r}^{K}: L^{2}(K) \rightarrow P_{r-1}(K)$ fulfil

$$
\begin{aligned}
\left|u-I_{r}^{K} u\right|_{1, K} \leq C h^{m}|u|_{m+1, K}, & & u \in H^{m+1}(K), m=0, \ldots, r \\
\left\|p-J_{r}^{K} p\right\|_{0, K} \leq C h^{\ell}|p|_{\ell, K}, & & p \in H^{\ell}(K), \ell=0, \ldots, r
\end{aligned}
$$

for all $K \in \mathcal{T}_{h}$. 
Proof. Exploiting the standard scaling properties of the affine reference mapping and its inverse, it suffices to show that we have the following estimates

$$
\begin{aligned}
\left|\hat{u}-\widehat{I_{r}^{K}} u\right|_{1, \widehat{K}} \leq C|\hat{u}|_{m+1, \widehat{K}}, & \hat{u} \in H^{m+1}(\widehat{K}), m=0, \ldots, r \\
\left\|\hat{p}-\widehat{J_{r}^{K}} p\right\|_{0, \widehat{K}} \leq C|\hat{p}|_{\ell, \widehat{K}}, \quad & \hat{p} \in H^{\ell}(\widehat{K}), \ell=0, \ldots, r
\end{aligned}
$$

on the corresponding reference cell $\widehat{K}$. We get

$$
\widehat{I_{r}^{K} u}=\widehat{I}_{r} \hat{u}, \quad \widehat{J_{r}^{K}} q=\widehat{J}_{r} \hat{q}
$$

from (26). Let $\widehat{E}$ be an arbitrary side of $\widehat{K}$ and $\widehat{N}^{S}$ an arbitrary nodal functional associated with $\widehat{E}$. Then, the continuity of the trace operator $\left.\hat{u} \mapsto \hat{u}\right|_{\widehat{E}}$ gives

$$
\left|\widehat{N}^{S}(\hat{u})\right| \leq C\|\hat{u}\|_{0, \widehat{E}} \leq C\|\hat{u}\|_{1, \widehat{K}}
$$

For an arbitrary nodal functionals $\widehat{N}^{C}$ which is associated with the interior of the reference cell $\widehat{K}$, we get

$$
\left|\widehat{N}^{C}(\hat{u})\right| \leq C\|\hat{u}\|_{0, \widehat{K}} \leq C\|\hat{u}\|_{1, \widehat{K}}
$$

We obtain together with $(25)$

$$
\left|\widehat{I}_{r} \hat{u}\right|_{1, \widehat{K}} \leq \sum_{i=1}^{n}\left|\widehat{N}_{i}(\hat{u}) \hat{\varphi}_{i}\right| \leq C\|\hat{u}\|_{1, \widehat{K}}
$$

which results in

$$
\left|\hat{u}-\widehat{I}_{r} \hat{u}\right|_{1, \widehat{K}} \leq C\|\hat{u}\|_{1, \widehat{K}} \leq C\|\hat{u}\|_{m+1, \widehat{K}}, \quad m \geq 0 .
$$

Hence, the mapping $\hat{u} \mapsto\left(\hat{u}-\widehat{I}_{r} \hat{u}\right)$ is continuous on $H^{m+1}(\widehat{K}), m \geq 0$. Moreover, the unisolvence ensures that $\hat{q}-\widehat{I}_{r} \hat{q}$ vanishes for $\hat{q} \in P_{m}(\widehat{K}), m=0, \ldots, r$, since $P_{r}(\widehat{K}) \subset \widehat{V}_{r}$. We get by using the Bramble-Hilbert lemma [3] the desired estimate

$$
\left|\hat{u}-\widehat{I_{r}^{K}} u\right|_{1, \widehat{K}}=\left|\hat{u}-\widehat{I}_{r} \hat{u}\right|_{1, \widehat{K}} \leq C|\hat{u}|_{m+1, \widehat{K}}, \quad 0 \leq m \leq r .
$$

The second estimate of this lemma follows in the same way.

We define global interpolation operators

$$
I_{r}: V_{g} \rightarrow V_{g h}, \quad J_{r}: Q \rightarrow Q_{h},
$$

by setting

$$
\left.\left(I_{r} v\right)\right|_{K}=I_{r}^{K} v,\left.\quad\left(J_{r} q\right)\right|_{K}=J_{r}^{K} q
$$

Obviously, $J_{r}$ maps into $Q_{h}$ since no continuity is required. We observe for $I_{r}$ that the definition (25) of the interpolation operator $\widehat{I}_{r}$ yields

$$
\left\langle\widehat{I_{r}} \hat{v}, \hat{q}\right\rangle_{\widehat{E}}=\langle\hat{v}, \hat{q}\rangle_{\widehat{E}} \quad \forall \hat{q} \in P_{r-1}(\widehat{E})
$$

for all sides $\widehat{E}$ of $\widehat{K}$. Here, $\langle\cdot, \cdot\rangle_{\widehat{E}}$ denotes the inner product in $L^{2}(\widehat{E})$. Since we consider only affine reference mappings, property (32) is transformed into

$$
\left\langle I_{r}^{K} v, q\right\rangle_{E}=\langle v, q\rangle_{E} \quad \forall q \in P_{r-1}(E)
$$


for all sides $E \in \mathcal{S}_{h}$ and all cell $K$ which have $E$ as one of their sides. Hence, the setting (31) maps into $V_{g h}$, see definition (24). Clearly, the component-wise defined vector-valued interpolation operator $\boldsymbol{I}_{r}^{K}$ inherits the properties from the $I_{r}^{K}$.

Using Lemma 5.1 and the fact that

$$
\left|\boldsymbol{v}-\boldsymbol{I}_{r} \boldsymbol{v}\right|_{1, h}^{2}=\sum_{K \in \mathcal{T}_{h}}\left|\boldsymbol{v}-\boldsymbol{I}_{r}^{K} \boldsymbol{v}\right|_{1, K}^{2}
$$

holds true, we get the following.

Corollary 5.2. The estimates

$$
\begin{aligned}
& \left|\boldsymbol{v}-\boldsymbol{I}_{r} \boldsymbol{v}\right|_{1, h} \leq C h^{m}|\boldsymbol{v}|_{m+1}, \quad \boldsymbol{v} \in \boldsymbol{H}^{m+1}(\Omega), \quad m=0, \ldots, r, \\
& \left\|q-J_{r} q\right\|_{0} \leq C h^{m}|q|_{m}, \quad q \in H^{m}(\Omega), \quad m=0, \ldots, r,
\end{aligned}
$$

are fulfilled for the global interpolation operators $\boldsymbol{I}_{r}$ and $J_{r}$.

Finally, we show that the vector-valued version $\boldsymbol{V}_{\boldsymbol{g} h}$ of nonconforming finite elements of order $r$ satisfies the inf-sup stability condition (H2) together with a discontinuous piecewise $P_{r-1}$ pressure.

Theorem 5.3. Let $\boldsymbol{V}_{\boldsymbol{g} h}$ be the vector-valued space which is derived from the scalar finite element space $V_{g h}$ of nonconforming finite elements of order $r$, see (24). Then, the finite element pair $\boldsymbol{V}_{\boldsymbol{g} h} / P_{r-1}^{\text {disc }}$ fulfils the inf-sup condition (H2).

Proof. Due to Fortin [8], the inf-sup condition (H2) is equivalent to the existence of an interpolation operator $\boldsymbol{i}_{h}: \boldsymbol{H}_{0}^{1}(\Omega) \rightarrow \boldsymbol{V}_{\mathbf{0} h}$ which satisfies

$$
\begin{aligned}
\left(\operatorname{div}_{\mathrm{h}} \boldsymbol{i}_{h} \boldsymbol{v}, q_{h}\right) & =\left(\operatorname{div} \boldsymbol{v}, q_{h}\right) & & \forall q \in Q_{h}, \boldsymbol{v} \in \boldsymbol{H}_{0}^{1}(\Omega), \\
\left|\boldsymbol{i}_{h} \boldsymbol{v}\right|_{1, h} & \leq C|\boldsymbol{v}|_{1}, & & \forall \boldsymbol{v} \in \boldsymbol{H}_{0}^{1}(\Omega),
\end{aligned}
$$

where the constant $C$ is independent of $h$. We will show that our interpolation operator $\boldsymbol{I}_{r}$ can be used as $\boldsymbol{i}_{h}$. The condition (33) is checked easily. We have

$$
\begin{aligned}
\left(\operatorname{div}_{\mathrm{h}} \boldsymbol{I}_{r} \boldsymbol{v}, q_{h}\right)=\sum_{K \in \mathcal{T}_{h}}\left(\operatorname{div} \boldsymbol{I}_{r}^{K} \boldsymbol{v}, q_{h}\right)_{K} & =\sum_{K \in \mathcal{T}_{h}}\left(-\left(\boldsymbol{I}_{r}^{K} \boldsymbol{v}, \nabla q_{h}\right)_{K}+\sum_{E \subset \partial K}\left\langle\boldsymbol{I}_{r}^{K} \boldsymbol{v} \cdot \boldsymbol{n}_{K}, q_{h}\right\rangle_{E}\right) \\
& =\sum_{K \in \mathcal{T}_{h}}\left(-\left(\boldsymbol{v}, \nabla q_{h}\right)_{K}+\sum_{E \subset \partial K}\left\langle\boldsymbol{v} \cdot \boldsymbol{n}_{K}, q_{h}\right\rangle_{E}\right)=\left(\operatorname{div} \boldsymbol{v}, q_{h}\right)
\end{aligned}
$$

where $\langle\cdot, \cdot\rangle_{\partial K}$ denotes the inner product of $L^{2}(\partial K)$. Furthermore, we have used integration by parts and the fact that the restriction of $q_{h} \in Q_{h}$ to any side $E$ belongs to $P_{r-1}(E)$ and that $\left.\nabla q_{h}\right|_{K} \in\left(P_{r-2}(K)\right)^{d}$. Furthermore, the definition of the nodal functionals was exploited.

The condition (34) follows immediately from Corollary 5.2 for $m=0$.

All four families of nonconforming finite elements together with suitable discontinuous pressure spaces fulfil the conditions (H1), (H2), and (H3). Hence, optimal convergence orders for the Stokes problem (1) are guaranteed, see [6].

\section{NumERICAL RESUlts}

This section presents some numerical results which were obtained by using the proposed nonconforming finite element spaces. All numerical computations have used the code MooNMD [14] and were carried out on a Linux PC (Pentium IV, $2.8 \mathrm{GHz})$. 
Since the solver aspects are not in the focus of this paper, we give here just a few notes on the solver, for details see [12]. A more detailed numerical study on the properties of solvers for the considered nonconforming discretisations of the Stokes problem will be subject of a forthcoming paper.

The discrete problem (6) is equivalent to a system of linear equations of the following form

$$
\left(\begin{array}{cc}
A & B^{T} \\
B & 0
\end{array}\right)\left(\begin{array}{l}
\underline{\mathbf{u}} \\
\underline{p}
\end{array}\right)=\left(\begin{array}{l}
\underline{F} \\
\underline{G}
\end{array}\right)
$$

where $\underline{\mathbf{u}}$ and $\underline{p}$ are the vector representations of $\mathbf{u}$ and $p$ with respect to fixed bases. The matrix $A$ is a $d \times d$ block-matrix with vanishing off-diagonal blocks and identical diagonal blocks. Hence, to store the matrix A, it suffices to store just one of the $d$ identical diagonal blocks. The matrix $B$ is a $1 \times d$ block-matrix where all $d$ blocks have to be stored since they are different.

The discrete systems of form (35) were solved by a geometric multigrid method which is based on general transfer operators introduced in [20] and Vanka-type smoothers [13,21]. Inside the Vanka-type smoother, the local systems for discretisations with a discontinuous pressure approximation consist of all degrees of freedom which are connected to a cell. For discretisations with continuous pressure, the local set of degrees of freedom contains exactly one pressure node and all velocity degrees of freedom which are coupled to this pressure node. Due to this, the discretised Stokes equations can be solved much faster for discretisations with a discontinuous pressure approximation since the smoothing costs are much smaller. The convergence of the used multilevel approach for the non-nested finite element spaces has been investigated in [15].

Concerning the conditioning of the arising discrete systems of form (35), the numerical calculations showed that the above described multigrid method works well also for the proposed nonconforming finite elements. The multigrid rates for the nonconforming elements are better than those for conforming discretisations with continuous pressure spaces but a little worse compared to conforming discretisations with discontinuous pressure approximations. For all $3 \mathrm{~d}$ calculations and for higher order discretisations in $2 \mathrm{~d}$, the total solver time is smallest for the nonconforming finite elements due to the smaller number of non-zero matrix entries. For all three types of discretisations (nonconforming and conforming with continuous and discontinuous pressure approximations), the used multigrid methods showed level-independent convergence rates.

\subsection{Two-dimensional problem}

Let $\Omega=(0,1)^{2}$. We choose $\boldsymbol{f}$ and $\boldsymbol{g}$ in the Stokes problem (1) such that

$$
\begin{aligned}
& \boldsymbol{u}(x, y)=\left(\begin{array}{c}
\sin x \sin y \\
\cos x \cos y
\end{array}\right), \\
& p(x, y)=2 \cos (x) \sin (y)-2 \sin (1)(1-\cos (1))
\end{aligned}
$$

is the solution. This example was taken from [2].

Table 1 shows the number of degrees of freedom for the two velocity components and the pressure for different types of conforming and nonconforming discretisations of the Stokes problem (1) on mesh level 6 which was obtained by 5 red refinement steps starting from a decomposition of the unit square into four congruent squares. Furthermore, Table 1 gives also the total number of non-zero matrix entries for storing the discrete system of form (35). As pointed out after (35), this includes the storage for just one of the two identical diagonal blocks of $A$ and the two blocks of $B$.

Since the difference between the results for the nonconforming families are very small, only the results for $V_{r}^{1} / P_{r-1}^{\text {disc }}$ are given. Table 2 shows for different discretisations the errors between the solution of the continuous problem and the discrete solutions on refinement level 6 . The given orders of convergence were obtained by using the results on refinement level 5 and 6 . The differences between discretisations with discontinuous pressure approximation for the same approximation order are quite small while the corresponding discretisation with a continuous pressure approximation gives for this example slightly better results. 
TABLE 1. Number of degrees of freedom and total number of non-zero matrix entries for different element pairs on level 6 .

\begin{tabular}{lrrrrrrr}
\hline Pair & $Q_{1}^{\text {rot }} / P_{0}$ & $Q_{2} / Q_{1}$ & $Q_{2} / P_{1}^{\text {disc }}$ & $V_{2} / P_{1}^{\text {disc }}$ & $Q_{3} / Q_{2}$ & $Q_{3} / P_{2}^{\text {disc }}$ & $V_{3} / P_{2}^{\text {disc }}$ \\
\hline Velocity & 16640 & 33282 & 33282 & 41472 & 74498 & 74498 & 74496 \\
Pressure & 4096 & 4225 & 12288 & 12288 & 16641 & 24576 & 24576 \\
Total & 20736 & 37507 & 45570 & 53760 & 91139 & 99074 & 99072 \\
\hline Matrix entries & 89472 & 463506 & 478745 & 516608 & 1903027 & 1695409 & 1575552 \\
\hline
\end{tabular}

TABLE 2. Errors and convergence orders on level 6 .

\begin{tabular}{ccccccc}
\hline & \multicolumn{2}{c}{$\left|\mathbf{u}-\mathbf{u}_{h}\right|_{1, h}$} & \multicolumn{2}{c}{$\left\|\mathbf{u}-\mathbf{u}_{h}\right\|_{0}$} & \multicolumn{2}{c}{$\left\|p-p_{h}\right\|_{0}$} \\
Element pair & Error & Order & Error & Order & Error & Order \\
\hline$Q_{1}^{\text {rot }} / P_{0}$ & $8.580-03$ & 0.99 & $2.598-05$ & 1.99 & $7.012-03$ & 1.00 \\
\hline$Q_{2} / Q_{1}$ & $8.104-06$ & 2.00 & $1.953-08$ & 3.00 & $1.146-05$ & 2.00 \\
$Q_{2} / P_{1}^{\text {disc }}$ & $8.106-06$ & 2.00 & $1.954-08$ & 3.00 & $2.144-05$ & 2.00 \\
$V_{2}^{1} / P_{1}^{\text {disc }}$ & $2.475-05$ & 1.98 & $4.732-08$ & 2.99 & $2.147-05$ & 2.00 \\
\hline$Q_{3} / Q_{2}$ & $3.109-08$ & 2.98 & $5.173-11$ & 3.97 & $3.382-08$ & 2.98 \\
$Q_{3} / P_{2}^{\text {disc }}$ & $5.977-08$ & 2.98 & $9.314-11$ & 3.99 & $6.660-08$ & 3.00 \\
$V_{3}^{1} / P_{2}^{\text {disc }}$ & $6.990-08$ & 2.99 & $1.117-10$ & 3.99 & $6.655-08$ & 3.00 \\
\hline
\end{tabular}

Let us consider the number of unknowns for each velocity component on a regular $N \times N$ decomposition of the unit square. In this case, we have $N^{2}$ vertices, $2 N^{2}$ edges, and $N^{2}$ cells. Note that we take only the highest power of $N$ into account. Considering a conforming discretisation with $Q_{r}$-elements, we have that each vertex is associated with one degree of freedom which has $(2 r+1)^{2}$ couplings, each edge has $(r-1)$ degrees of freedom where each of them connects to $(2 r+1)(r+1)$ degrees of freedom, each cell has $(r-1)^{2}$ inner degrees of freedom which are connected to $(r+1)^{2}$ degrees of freedom. Hence, we have $N^{2} r^{2}$ degrees of freedom and approximately $N^{2} r^{4}$ non-zero entries (couplings) in the matrix $A$. This gives roughly $r^{2}$ for the averaged number of non-zero matrix entries per degree of freedom. Looking at a nonconforming discretisation, we have no degrees of freedom at the vertices, $r$ degrees per edge with $2 \operatorname{dim} \widehat{V}_{r}-r=r^{2}+6 r$ couplings, and $\operatorname{dim} P_{r-2}^{2 D}=r(r-1) / 2$ degrees of freedom in the cell interior with $\operatorname{dim} \widehat{V}_{r}=r(r+7) / 2$ couplings. Hence, we obtain $N^{2} r^{2} / 2$ degrees of freedom with a total number of approximately $N^{2} r^{4} / 4$ non-zero entries in the matrix $A$. This gives an average of $r^{2} / 2$ for the number of non-zero matrix entries per degree of freedom. Since for higher order discretisations the asymptotic number of degrees of freedom is determined by the number of degrees of freedom which are associated with the cell interior, we find that the main reason for the different behaviour of conforming and nonconforming discretisations is that there are $r^{2}$ degrees of freedom in the interior of each cell for conforming discretisations (corresponding to the dimension of $Q_{r-2}^{2 D}$ ) while there are just $r^{2} / 2$ interior degrees of freedom (corresponding to the dimension of $P_{r-2}^{2 D}$ ) for nonconforming methods. To summarise, we have for higher order discretisations just half the number of unknowns and only a quarter of the non-zero entries in the matrix $A$. For small values of $r$ as given in Table 1, both discretisation types have nearly the same number of unknowns while, even for the small value $r=3$, the number of non-zero matrix entries for the nonconforming discretisation is less than the number for the corresponding confirming methods. 
TABLE 3. Number of degrees of freedom and total number of non-zero matrix entries for different element pairs on level 4.

\begin{tabular}{lrrrrrrr}
\hline Pair & $Q_{1}^{\text {rot }} / P_{0}$ & $Q_{2} / Q_{1}$ & $Q_{2} / P_{1}^{\text {disc }}$ & $V_{2} / P_{1}^{\text {disc }}$ & $Q_{3} / Q_{2}$ & $Q_{3} / P_{2}^{\text {disc }}$ & $V_{3} / P_{2}^{\text {disc }}$ \\
\hline Velocity & 39168 & 107811 & 107811 & 129792 & 352947 & 352947 & 284160 \\
Pressure & 4096 & 4913 & 16384 & 16384 & 35937 & 40960 & 40960 \\
Total & 43264 & 112724 & 124195 & 146176 & 388884 & 393907 & 325120 \\
\hline Matrix entries & 201984 & 3461336 & 3194117 & 2225920 & 29298862 & 20527483 & 10694656 \\
\hline
\end{tabular}

TABLE 4. Errors and convergence orders on level 4.

\begin{tabular}{ccccccc}
\hline & \multicolumn{2}{c}{$\left|\mathbf{u}-\mathbf{u}_{h}\right|_{1, h}$} & \multicolumn{2}{c}{$\left\|\mathbf{u}-\mathbf{u}_{h}\right\|_{0}$} & \multicolumn{2}{c}{$\left\|p-p_{h}\right\|_{0}$} \\
Element pair & Error & Order & Error & Order & Error & Order \\
\hline$Q_{1}^{\text {rot }} / P_{0}$ & $5.210-01$ & 0.99 & $6.634-03$ & 1.97 & $8.241-02$ & 1.05 \\
\hline$Q_{2} / Q_{1}$ & $6.773-03$ & 2.00 & $6.536-05$ & 3.00 & $1.699-03$ & 2.03 \\
$Q_{2} / P_{1}^{\text {disc }}$ & $6.772-03$ & 2.00 & $6.531-05$ & 3.00 & $1.934-03$ & 2.00 \\
$V_{2} / P_{1}^{\text {disc }}$ & $1.616-02$ & 1.99 & $1.631-04$ & 2.99 & $1.963-03$ & 2.06 \\
\hline$Q_{3} / Q_{2}$ & $1.158-04$ & 2.98 & $8.491-07$ & 3.95 & $6.006-05$ & 2.94 \\
$Q_{3} / P_{2}^{\text {disc }}$ & $1.084-04$ & 2.99 & $7.793-07$ & 3.98 & $4.561-05$ & 3.00 \\
$V_{3} / P_{2}^{\text {disc }}$ & $5.500-04$ & 2.98 & $4.008-06$ & 3.98 & $4.634-05$ & 3.03 \\
\hline
\end{tabular}

\subsection{Three-dimensional problem}

Let $\Omega=(0,1)^{3}$. We have chosen $\boldsymbol{f}$ and $\boldsymbol{g}$ in the Stokes problem (1) such that

$$
\begin{aligned}
& \boldsymbol{u}(x, y, z)=\left(\begin{array}{c}
\sin (\pi x) \sin (\pi y) \sin (\pi z)+x^{4} \cos (\pi y) \\
\cos (\pi x) \cos (\pi y) \cos (\pi z)-3 y^{3} z \\
\cos (\pi x) \sin (\pi y) \cos (\pi z)+\cos (\pi x) \sin (\pi y) \sin (\pi z)-4 x^{3} \cos (\pi y) z+4.5 y^{2} z^{2}
\end{array}\right) \\
& p(x, y, z)=3 x-\sin (y+4 z)-p_{0}
\end{aligned}
$$

is the unique solution of (1) where the constant $p_{0}$ is determined by $p \in L_{0}^{2}(\Omega)$.

Table 3 shows the number of degrees of freedom for the three velocity components and the pressure for different types of conforming and nonconforming discretisations of the Stokes problem (1) on mesh level 4 which was obtained by 3 red refinement steps starting from a decomposition of the unit cube into eight congruent cubes. Moreover, Table 3 presents also the total number of non-zero matrix entries for storing the discrete system of form (35). As pointed out after (35), this includes the storage for just one of the three identical diagonal blocks of $A$ and the three blocks of $B$.

Table 4 shows for different discretisations the errors between the solution of the continuous problem and the discrete solutions on refinement level 4. The given orders of convergence were obtained by using the results on refinement level 3 and 4 . We clearly see that all discretisations converge with optimal orders. Moreover, the results for all discretisations with the same approximation order are comparable.

Let us consider the number of unknowns for each velocity component on a regular $N \times N \times N$ decomposition of the unit cube. In this case, we have $N^{3}$ vertices, $3 N^{3}$ edges, $3 N^{3}$ faces, and $N^{3}$ cells. Note again that we take only the highest power of $N$ into account. Considering a conforming discretisation with $Q_{r}$-elements, we have that each vertex is associated with one degree of freedom which has $(2 r+1)^{3}$ couplings, each edge has $(r-1)$ degrees of freedom where each of them connects to $(2 r+1)^{2}(r+1)$ degrees of freedom, each face has $(r-1)^{2}$ degrees of freedom with $(2 r+1)(r+1)^{2}$ couplings for each of them, each cell has $(r-1)^{3}$ inner degrees of freedom which are connected to $(r+1)^{3}$ degrees of freedom. Hence, we have $N^{3} r^{3}$ degrees of freedom and approximately $N^{3} r^{6}$ non-zero entries in the matrix $A$. This gives roughly $r^{3}$ for the averaged number of non-zero matrix entries per degree of freedom. Looking at a nonconforming discretisation, we have no degrees of freedom at the vertices 
and the edges, $\operatorname{dim} P_{r-1}^{2 D}=r(r+1) / 2$ degrees per face with $2 \operatorname{dim} \widehat{V}_{r}-r(r+1) / 2=r(r+1)(2 r+31) / 6$ couplings, and $\operatorname{dim} P_{r-2}^{3 D}=(r+1) r(r-1) / 6$ degrees of freedom in the cell interior with $\operatorname{dim} \widehat{V}_{r}=r(r+1)(r+17) / 6$ couplings. Hence, we obtain $N^{3} r^{3} / 6$ degrees of freedom with a total number of approximately $N^{3} r^{6} / 36$ nonzero entries in the matrix $A$. This gives an average of $r^{3} / 6$ for the number of non-zero matrix entries per degree of freedom. Even for the small values $r=3$, the nonconforming discretisation has less degrees of freedom and much less non-zero matrix entries, see Table 3. The main reason for the different behaviour of conforming and nonconforming discretisations is that there are approximately $r^{3}$ inner degrees of freedom per cell (corresponding to the dimension of $Q_{r-2}^{3 D}$ ) for conforming discretisations compared to approximately $r^{3} / 6$ inner degrees of freedom (corresponding to the dimension of $P_{r-2}^{3 D}$ ) for nonconforming methods.

\section{Conclusions}

We have considered in this paper inf-sup stable nonconforming discretisation of higher order for the Stokes problem. Starting from the general convergence theory given in [6], we considered three families of nonconforming elements on quadrilaterals and one family on hexahedra. Moreover, the unisolvence of two novel families with respect to the set of nodal functionals was proved. All four families fulfil the conditions (H1), (H2), and (H3). Hence, optimal orders of convergence are guaranteed. The numerical study in Section 6 shows on the one hand the optimal orders of convergence. Moreover, the errors obtained by the nonconforming discretisation and the errors for conforming discretisations are comparable. On the other hand, nonconforming discretisations of higher order, especially in 3D, need less unknowns and, even more important, much less non-zero matrix entries. This shows the potential of nonconforming discretisations of higher order.

\section{REFERENCES}

[1] M. Bercovier and O. Pironneau, Error estimates for finite element method solution of the Stokes problem in the primitive variables. Numer. Math. 33 (1979) 211-224.

[2] D. Braess and R. Sarazin, An efficient smoother for the Stokes problem. Appl. Numer. Math. 23 (1997) 3-19.

[3] J.H. Bramble and S.R. Hilbert, Estimation of linear functionals on Sobolev spaces with application to Fourier transforms and spline interpolation. SIAM J. Numer. Anal. 7 (1970) 112-124.

[4] Z. Cai, J. Douglas, Jr. and X. Ye, A stable nonconforming quadrilateral finite element method for the stationary Stokes and Navier-Stokes equations. Calcolo 36 (1999) 215-232.

[5] Z. Cai, J. Douglas, Jr., J.E. Santos, D. Sheen and X. Ye, Nonconforming quadrilateral finite elements: a correction. Calcolo 37 (2000) 253-254.

[6] M. Crouzeix and P.-A. Raviart, Conforming and nonconforming finite element methods for solving the stationary Stokes equations I. RAIRO. Anal. Numér. 7 (1973) 33-76.

[7] J. Douglas, Jr., J.E. Santos, D. Sheen and X. Ye, Nonconforming Galerkin methods based on quadrilateral elements for second order elliptic problems. ESAIM: M2AN 33 (1999) 747-770.

[8] M. Fortin, An analysis of the convergence of mixed finite element methods. RAIRO Anal. Numér. 11 (1977) 341-354.

[9] V. Girault and P.-A. Raviart, Finite Element Methods for Navier-Stokes equations. Springer-Verlag, Berlin-Heidelberg-New York (1986).

[10] H.D. Han, Nonconforming elements in the mixed finite element method. J. Comput. Math. 2 (1984) 223-233.

[11] J.P. Hennart, J. Jaffré and J.E. Roberts, A constructive method for deriving finite elements of nodal type. Numer. Math. 53 (1988) 701-738.

[12] V. John, Large Eddy Simulation of Turbulent Incompressible Flows. Analytical and Numerical Results for a Class of LES Models. Lecture Notes in Computational Science and Engineering 34, Springer-Verlag, Berlin, Heidelberg, New York (2003).

[13] V. John and G. Matthies, Higher-order finite element discretizations in a benchmark problem for incompressible flows. Int. J. Num. Meth. Fluids 37 (2001) 885-903.

[14] V. John and G. Matthies, MooNMD - a program package based on mapped finite element methods. Comput. Vis. Sci. 6 (2004) $163-169$.

[15] V. John, P. Knobloch, G. Matthies and L. Tobiska, Non-nested multi-level solvers for finite element discretisations of mixed problems. Computing 68 (2002) 313-341.

[16] G. Matthies and L. Tobiska, The inf-sup condition for the mapped $Q_{k} / P_{k-1}^{\text {disc }}$ element in arbitrary space dimensions. Computing 69 (2002) 119-139.

[17] G. Matthies and L. Tobiska, Inf-sup stable non-conforming finite elements of arbitrary order on triangles. Numer. Math. 102 (2005) 293-309. 
[18] J. Maubach and P. Rabier, Nonconforming finite elements of arbitrary degree over triangles. RANA report 0328, Technical University of Eindhoven (2003).

[19] R. Rannacher and S. Turek, Simple nonconforming quadrilateral Stokes element. Numer. Meth. Part. Diff. Equ. 8 (1992) 97-111.

[20] F. Schieweck, A general transfer operator for arbitrary finite element spaces. Preprint 00-25, Fakultät für Mathematik, Ottovon-Guericke-Universität Magdeburg (2000).

[21] S. Vanka, Block-implicit multigrid calculation of two-dimensional recirculating flows. Comp. Meth. Appl. Mech. Engrg. 59 (1986) 29-48.

[22] R. Verfürth, Error estimates for a mixed finite element approximation of the Stokes equations. RAIRO Anal. Numér. 18 (1984) $175-182$. 